\title{
Reconfigurable 26GHz Liquid Metal Antenna Capable of Low Loss Continuous Beam Steering
}

\author{
James R Kelly \\ ICS \\ University of Surrey \\ Guildford, UK \\ j.r.kelly@surrey.ac.uk
}

\author{
Mandana Ardeshir Tanha \\ ICS \\ University of Surrey \\ Guildford, UK \\ m.ardeshirtanha@surrey.ac.uk
}

\begin{abstract}
For this first time this paper presents a concept for a high gain millimeter wave beam steerable antenna that supports continuous steering over a $360^{\circ}$ scan range and promises to yield extremely low power losses. The concept does not require phase shifters which suffer from high insertion losses at millimeter wave frequencies or mechanical movement, in the conventional sense, which has the disadvantages of poor reliability and slow response times due to high inertia.
\end{abstract}

Keywords-Millimeter wave antenna, beamsteering, beamforming, steerable antenna, Yagi-Uda antenna, liquid metal, EGaIn, reconfigurable antenna

\section{INTRODUCTION}

There is currently significant interest in using millimetre wave frequencies for next generation mobile communication applications. At these frequencies the path loss is high, necessitating the use of high gain antennas. A total antenna gain of $37 \mathrm{dBi}$ is projected to be sufficient in the link budget to maintain a reliable mm-wave radio link [1]. Where mobility is required beamsteering is also important. Applications such as backhaul and access require continuous beamsteering over a wide scan angle range. There are currently only a handful of techniques by which this can be achieved, including: mechanical movement [2] and phased array approaches [3]. Unfortunately phase shifters for millimetre wave frequencies are currently only available up to frequencies of about $40 \mathrm{GHz}$. Furthermore commercially available millimetre wave digital phase shifters exhibit very high levels of insertion loss, on the order of $16 \mathrm{~dB}$ for a 26 $\mathrm{GHz}$ phase shifter. Continuously variable phase shifters would be required for continuous beam steering. Unfortunately these exhibit even higher levels of insertion loss. This makes the technology very power hungry and energy inefficient. For these reasons we would like to design antennas that do not involve phase shifters.

In this paper we propose a Yagi-Uda antenna that is based closely on the design reported in [4]. The antenna is capable of producing a single high gain beam that can be continuously steered in the azimuthal plane. This is achieved by employing a reflector, along with a series of directors. These parasitic elements are formed from liquid metal and would be contained within a cylindrical PDMS microfluidic channel structure. In this way the main beam of the antenna could be steered by rotating both sets of elements through the angle labelled "Angle 2" in Fig. 1. The entire structure is made in a cylindrical format thus preserving symmetry.

The proposed antenna is a valuable alternative to a phased array. But equally it could be employed as the radiating element within a phased array. This would enable one to implement the approach of element factor plus array factor control [3] which has been shown to yield valuable improvements in the scan range and average gain over the scan angle range. With the aid of the proposed antenna, one would be able to steer the element factor in a continuous fashion. This would enable the peak of the gain versus scan angle curve to be moved to the achieved scan angle. This is expected to yield a significant reduction in scan loss.

We envisage that the proposed antenna could find applications in millimetre wave small cell base stations, particularly for indoor access points. The antenna is also a viable candidate for backhaul applications. Before the antenna could be employed in outdoor base station applications it would require further development. For example, it would be necessary to increase the gain, provide for dual polarisation, and support multiple users etc.

\section{RESULTS}

This section of the paper presents results which demonstrate the feasibility of the proposed concept. Further work is needed to refine the results. Fig. 1 shows the structure of the 5 element Yagi-Uda antenna. Table 1 lists the dimensions of the antenna. The antenna has been designed for operation at $26 \mathrm{GHz}$. The parasitic elements in the antenna are all formed from a commercially available liquid metal known as EGaIn having a conductivity of $3.4 \times 10^{6}$.

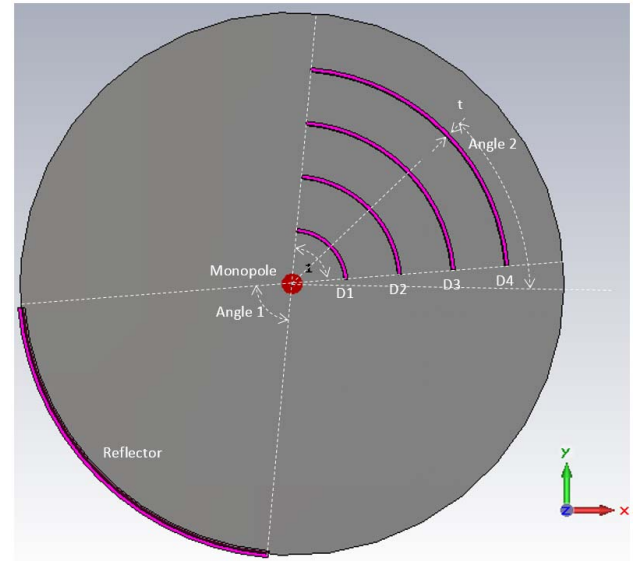

(a)

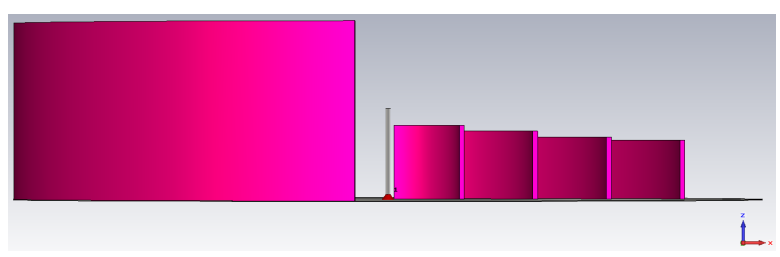

(b)

Fig. 1. Sturcture of the 5 element Yagi-Uda antenna. (a) top view, (b) side view. 
TABLE I.

DiMENSIONS OF THE ANTENNA

\begin{tabular}{|c|c|}
\hline Parameter & Dimension \\
\hline Radius of ground plane & $2 \lambda_{0}(23 \mathrm{~mm})$ \\
\hline Height of reflector & $0.475 \lambda_{0}(5.463 \mathrm{~mm})$ \\
\hline Height of monopole & $0.22 \lambda_{0}(2.53 \mathrm{~mm})$ \\
\hline Height of D1 & $0.2 \lambda_{0}(2.3 \mathrm{~mm})$ \\
\hline Height of D2 & $0.184 \lambda_{0}(2.116 \mathrm{~mm})$ \\
\hline Height of D3 & $0.168 \lambda_{0}(1.932 \mathrm{~mm})$ \\
\hline Height of D4 & $0.16 \lambda_{0}(1.84 \mathrm{~mm})$ \\
\hline Angle 1 & $80^{\circ}$ \\
\hline Angle 2 & $35^{\circ}$ \\
\hline Separation between the directors & $0.2 \lambda_{0}(2.3 \mathrm{~mm})$ \\
\hline
\end{tabular}

Fig. 2 shows the return loss performance of the antenna. The antenna exhibits a $10 \mathrm{~dB}$ return loss bandwidth of 2.98 $\mathrm{GHz}$.

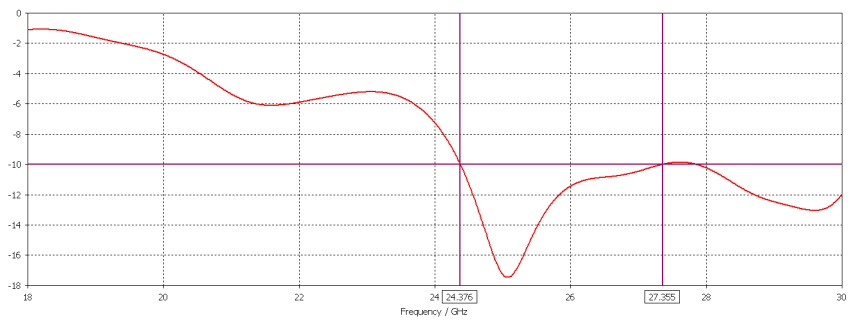

Fig. 2. Return loss performance of the antenna.

Fig. 2 shows the radiation pattern for the 5 element antenna shown in Fig. 1. The driven element of the antenna is a wire molopole. The directivity of the monopole alone is $3.88 \mathrm{dBi}$. The complete antenna exhibits a directivity of 7.11 $\mathrm{dBi}$. A well known rule of thum states that the directivity of a Yagi Uda antenna can be approximated as follows:

$$
\mathrm{D}_{\mathrm{YU}}=\text { number of directors } \times \text { gain of driven element }
$$

The achieved directivity of the antenna is thus 0.73 of the expected value. The main beam, shown in Fig. 2, is aligned with a line bisecting the reflector and directors. Due to the symmetry of the structure the antenna exbitis the same directivity in all directions around the structure and thus does not suffer from scan loss. Further refinements are needed to the antenna to meet the demands of the application requirements. For example, the antenna currently exhibits a high lobe level at off axis angles. The cause of this needs to be carefully and systematically explored. We speculate that the problem arrises due to the refection and difraction of energy from the directors which are quite large in their extent. The effect could probably be reduced by optimising the profile of the directors and/or through the use of absorber material placed in strategic locations. The relatively large size and close proximity of
D1 leads to difficulties in matching the antenna. This was overcome by tuning the diameter of the monopole wire. But the matching is very sensitive to this parameter which could lead to fabrication difficulties.

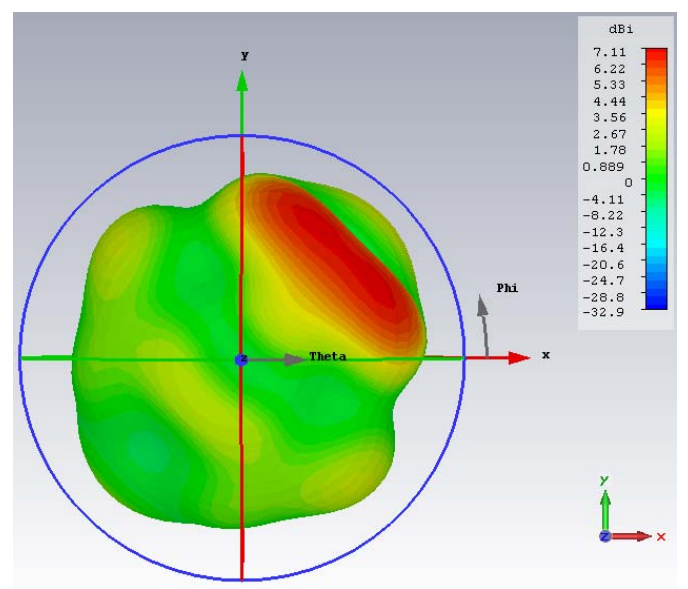

Fig. 3. Radiation pattern showing directivity achieved.

\section{CONCLUSION}

This paper presents a concept for an antenna which promises to enable continuous steering over a wide scan angle range with very loss insertion loss and no scan loss. The antenna relies on the use of liquid metal and the unique opertunities for new forms of antenna reconfiguration that it affords. The antenna is suitable for base station and backhaul applications. Further developments are required to improve the gain of the antenna to at least $18 \mathrm{dBi}$. For this reason a new version of the antenna will be developed incorporating a larger number of directors. If we were to employ 14 directors Eq. (1) predicts a directivity of $34 \mathrm{dBi}$. Even with the gain reduction observed above one would therefore expect to meet the directivity target. The overall size of a 14 element antenna would be around $57.5 \mathrm{~mm}$ i.e. approximately equal to the width of a standard smart phone handset.

\section{ACKNOWLEDGMENT}

The authors would like to acknowledge the support of EPSRC (EP/P008402/1) as well as the University of Surrey 5GIC (http://www.surrey.ac.uk/5gic) members for this work.

\section{REFERENCES}

[1] M. S. Rabbani, James Churm, Alexandros Feresidis, "Towards, Millimetre-wave Antenna Design for Future Mobile Broadband Networks," LAPC, IET, Loughborough, UK. 2017.

[2] Fast and low-cost beam steering using an agile mechanical feed system for exciting circular arrays, Ali Mirkamali; Ramin Deban; Francis Siaka; Jean-Jacques Laurin, IET Microwaves, Antennas \& Propagation, Year: 2016, Volume: 10, Issue: 4, Pages: 378 - 384.

[3] Wide-Angle Scanning Low Profile Phased Array Antenna Based on a Novel Magnetic Dipole, Chun-Mei Liu; Shao-Qiu Xiao; Hui-Lin Tu; Zhuofu Ding, IEEE Transactions on Antennas and Propagation, Year: 2017, Volume: 65, Issue: 3, Pages: $1151-1162$.

[4] Andy M. Morishita; Carolynn K. Y. Kitamura; Aaron T. Ohta; Wayne A. Shiroma, "A Liquid-Metal Monopole Array With Tunable Frequency, Gain, and Beam Steering," IEEE Antennas and Wireless Propagation Letters, Vol. 12, pp. 1388 - 1391, 2013. 